\title{
ACT domain of Bacillus anthracis prephenate dehydrogenase acts as tyrosine sensor and inhibits the enzyme via a mechanical switch
}

Ivan G. Shabalin ${ }^{1}$, Artyom Gritsunov², Jing Hou ${ }^{3}$, Joanna Lipowska ${ }^{4}$, Charles D. Miks ${ }^{5}$, David R. Cooper ${ }^{6}$, Wladek Minor ${ }^{7}$ and Dinesh Christendat ${ }^{8}$

${ }^{1}$ University of Virginia, Charlottesville, VA, USA, ivan s@iwonka.med.virginia.edu

2 University of Toronto, Toronto, Canada, artyom.gritsunov@mail.utoronto.ca

${ }^{3}$ University of Virginia, Charlottesville, VA, USA, houjing@iwonka.med.virginia.edu

${ }^{4}$ University of Virginia, Charlottesville, VA, USA, joanna I@iwonka.med.virginia.edu

${ }^{5}$ University of Virginia, Charlottesville, VA, USA, dylan m@iwonka.med.virginia.edu

${ }^{6}$ University of Virginia, Charlottesville, VA, USA, dcoop@iwonka.med.virginia.edu

7 University of Virginia, Charlottesville, VA, USA, wladek@iwonka.med.virginia.edu

${ }^{8}$ University of Toronto, Toronto, Canada, dinesh.christendat@utoronto.ca

Tyrosine biosynthesis via the shikimate pathway is absent in humans and other animals, making it an attractive target for next-generation antibiotics. Enzymes of tyrosine biosynthesis are also of interest for the "green" production of pharmaceuticals, aromatic polymers, and petrochemical aromatics. Prephenate dehydrogenase (PDH) catalyzes the penultimate step of tyrosine biosynthesis in bacteria, the NAD(P)+ dependent oxidative decarboxylation of prephenate to 4hydroxyphenylpyruvate. The majority of PDHs are dimers; each monomer consists of a nucleotide-binding domain and a dimerization domain, which together form the dimeric catalytic core (Fig. 1). Certain PDHs, including several from pathogens on the WHO priority list of antibioticresistant bacteria, possess an additional ACT domain, making them an attractive target in the war against antibiotic resistance. Previously, these enzymes have not been structurally and biochemically studied due to difficulties with protein aggregation.

We have established a recombinant protein expression system for PDH from Bacillus anthracis $(\mathrm{BaPDH})$, the causative agent of anthrax, and have conducted detailed kinetic and biophysical analyses of this enzyme. We also determined three structures of BaPDH: a ternary complex with $\mathrm{NAD}^{+}$and tyrosine, a binary complex with tyrosine, and a structure of an isolated ACT domain dimer. Our kinetic data shows that BaPDH is allosterically regulated by tyrosine. Our structural analysis revealed that tyrosine binds to the ACT domains of BaPDH dimer resulting in a conformational state of the enzyme that sterically prevents prephenate binding to the active site (Fig. 1). These findings demonstrate that the binding of tyrosine to the ACT domains allosterically inhibits BaPDH activity via a mechanical switch, which is modulated by the loop that links the ACT domain to the catalytic core and by the latch loop of the ACT domain. This mode of allosteric inhibition is novel compared to the competitive inhibition model established for other PDHs and is different than the mechanisms of other ACT-containing enzymes. This study provides new mechanistic insights that advance our understanding of tyrosine biosynthesis in bacteria.
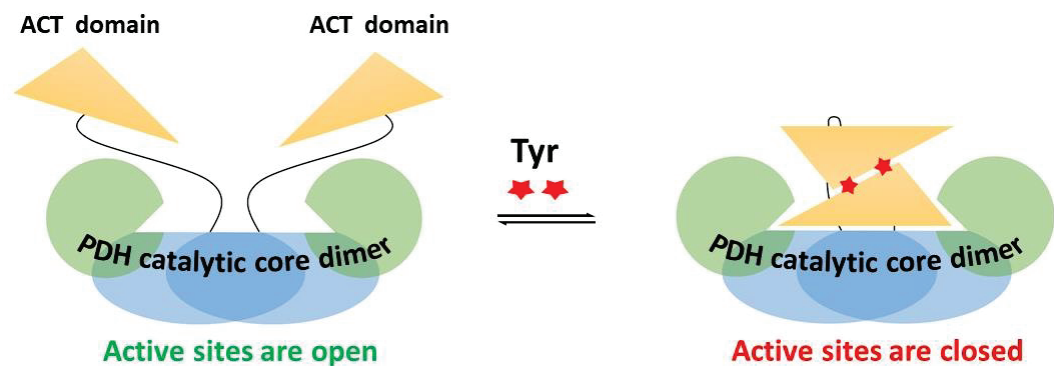

Fig. 1. The proposed mechanism of BaPDH inhibition 